\title{
"Latent Gastric Cancer" after Eradicating Helicobacter pylori
}

\author{
Yoshiharu Uno \\ Office Uno Column, Kakogawa, Japan
}

\section{To the Editor:}

I was very interested in the results of the study by Maehata et al. ${ }^{1}$ on metachronous gastric cancer (MGC) after eradicating Helicobacter pylori. MGC is a gastric cancer that occurs after endoscopic resection (ER) of primary early gastric cancer. Because gastric cancers detected within 1 year after ER should be regarded as a missed synchronous gastric cancer, MGC is defined as a new gastric cancer developing in areas other than the primary gastric cancer site at least 1 year after ER, while synchronous gastric cancer is defined as a second cancer found within 1 year after ER., ${ }^{1,2}$ Atrophy-free mucosa, atrophy and/or intestinal metaplasia can be found in the stomachs of $H$. pyloriinfected persons, but the mucosa that develops primary cancer is the riskiest background mucosa. Therefore, whether MGC can be prevented by eradicating $H$. pylori is one of the most crucial factors for justifying eradication therapy. In 2012, Maehata et $a{ }^{2}$ were the first to publish that during an 11-year observation study, MGC incidence increased 5 years after eradicating $H$. pylori. Their data showed that the cumulative incidence rate after 5 years was equivalent to the group that did not undergo $H$. pylori eradication. Therefore, I believe that the MGC became "latent gastric cancer (LGC)," which is difficult to detect endoscopically within 5 years after eradicating $H$. pylori.

The 2008 study by Fukase et al., ${ }^{3}$ which suggested that MGC could be prevented by eradicating $H$. pylori, only observed patients over a 3-year period. In 2014, Yoon et al., ${ }^{4}$ conducted a meta-analysis of 13 clinical studies and reported that eradicating $H$. pylori prevented MGC. However, the observation periods of these 13 studies ranged from 23 to 61 months. In other words, the meta-analysis of Yoon et al. covered only the first half of the observation period used in the study by Maehata et al. of 2012. Thus, many studies conducted within the 5-year observation period are objective and could evidence the existence of LGC. In a recent study, Maehata et al. ${ }^{1}$ investigated the reason that gastric cancer was undetected during the first half of the 11-year study period (shaded area of Fig. 1).

Maehata et al. found that the MGC lesions detected following successful eradication were small and depressed but with a high prevalence of lymphatic and venous invasion. In theory, after 5 years, it is possible that such MGC will develop to a more advanced stage compared with pure gastric cancer without eradication. Therefore, after eradication, detecting MGC within 5 years becomes important. However, such small MGC lesions are occasionally covered by non-neoplastic epithelium due to influences such as improved gastric acid secretion induced after eradicating $H$. pylori. ${ }^{1,5}$

This may be a new morphological cancer, artificially processed by eradicating $H$. pylori. In addition, LGC may occur in early gastric cancer patients treated by $\mathrm{ER}$, as well as in patients after $H$. pylori eradication with asymptomatic chronic gastritis. Regardless of MGC, gastric cancer after eradicating $H$. pylori is often difficult to diagnose endoscopically because of the indistinct border or disappearance of the characteristic tumor surface

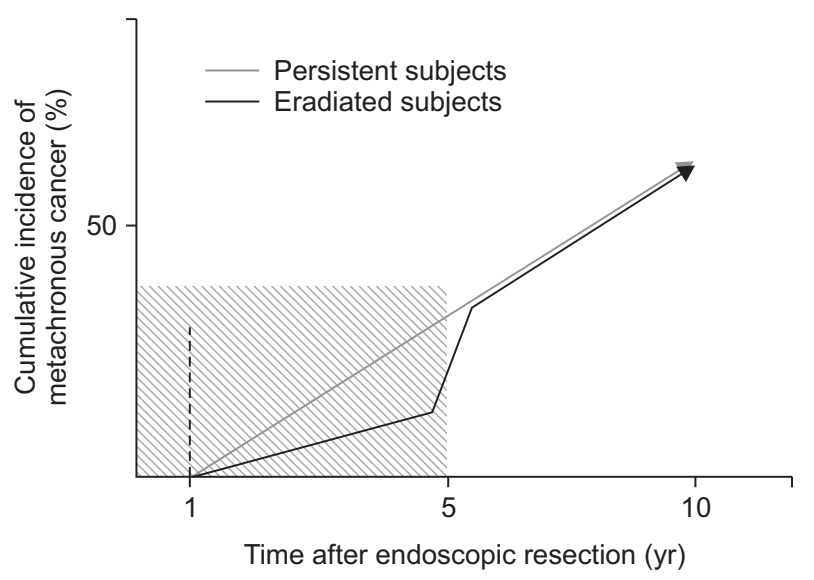

Fig. 1. The cumulative incidence of metachronous gastric cancer. Adapted from Maehata Y, et al. Gastrointest Endosc 2012;75:39-46. ${ }^{2}$

Correspondence to: Yoshiharu Uno

Office Uno Column, 419-2 Yota, Onoe-Cho, Kakogawa 675-0025, Japan

Tel: +81-79-439-4697, Fax: +81-79-439-4697, E-mail: yoshiharu333@hotmail.com

Received on October 20, 2017. Accepted on December 9, 2017.

pISSN 1976-2283 eISSN 2005-1212 https://doi.org/10.5009/gnl17463

(a) This is an Open Access article distributed under the terms of the Creative Commons Attribution Non-Commercial License (http://creativecommons.org/licenses/by-nc/4.0) which permits unrestricted non-commercial use, distribution, and reproduction in any medium, provided the original work is properly cited. 
structures. ${ }^{6}$ Furthermore, traditional endoscopic diagnostic methods for early gastric cancer, which have been studied and systematized in Japan for more than half a century, are based on the natural course of the $H$. pylori-infected stomach and might not be applicable for LGC. It is often difficult for endoscopists with average levels of training to detect such LGC. Therefore, it should be debated whether these patients should have $H$. pylori eradicated. Due to the hype surrounding this treatment, most patients who underwent $H$. pylori eradication may believe that their risk of cancer has decreased, thus they may not attend follow-up examinations. Indeed, five patients who did not undergo endoscopic follow-up recently died in Japan. ${ }^{5}$ These patients died from MGC more than 5 years after ER of the primary cancer. Therefore, I believe that the results of Maehata et al. ${ }^{1,2}$ should be made available to patients in Japan, Korea, and China who are commonly afflicted with these problems.

\section{CONFLICTS OF INTEREST}

No potential conflict of interest relevant to this article was reported.

\section{REFERENCES}

1. Maehata Y, Nakamura S, Esaki M, et al. Characteristics of primary and metachronous gastric cancers discovered after helicobacter pylori eradication: a multicenter propensity score-matched study. Gut Liver 2017;11:628-634.

2. Maehata Y, Nakamura S, Fujisawa K, et al. Long-term effect of Helicobacter pylori eradication on the development of metachronous gastric cancer after endoscopic resection of early gastric cancer. Gastrointest Endosc 2012;75:39-46.

3. Fukase K, Kato M, Kikuchi S, et al. Effect of eradication of Helicobacter pylori on incidence of metachronous gastric carcinoma after endoscopic resection of early gastric cancer: an open-label, randomised controlled trial. Lancet 2008;372:392-397.

4. Yoon SB, Park JM, Lim CH, Cho YK, Choi MG. Effect of Helicobacter pylori eradication on metachronous gastric cancer after endoscopic resection of gastric tumors: a meta-analysis. Helicobacter 2014;19:243-248.

5. Abe S, Oda I, Minagawa T, et al. Metachronous gastric cancer following curative endoscopic resection of early gastric cancer. Clin Endosc. Epub 2017 Sep 18. https://doi.org/10.5946/ce.2017.104.

6. Ohba R, Iijima K. Pathogenesis and risk factors for gastric cancer after Helicobacter pylori eradication. World J Gastrointest Oncol 2016;8:663-672. 